\title{
Anestesia para cirugía no cardíaca en pacientes pediátricos portadores de cardiopatías congénitas
}

\author{
2a Parte
}

SILVANA CAVALLIERI B. ${ }^{1}$, FRANCISCO BOYE R. ${ }^{2}$

\section{Coartación aórtica}

1 a Coartación de la Aorta (CoAo) representa un $5-8 \%$ de todas las cardiopatías congénitas. Consiste en una estenosis de la arteria aórtica en la zona cercana al ductus arterioso, lo que provoca una obstrucción al flujo sistémico del hemicuerpo inferior. La lesión en el neonato puede asociarse a otros haIlazgos como hipoplasia del arco de la aorta, DAP, estenosis del tracto de salida del VI (sub, supra valvular aortica, CIV o combinaciones). Cuando se detecta en niños mayores es habitualmente un defecto único o asociado a válvula aórtica bicúspide.

\section{Fisiopatología}

La consecuencia hemodinámica de la lesión en la aorta es una pobre perfusión distal a la obstrucción, particularmente en recién nacidos, donde la presentación es más grave y como se dijo, muchas veces asociada a otros defectos. En recién nacidos puede asociarse a hipoplasia del arco de la aorta o a una CoAo crítica, entonces se considera una patología ductus dependiente y el tratamiento inmediato es mantener el ductus permeable mediante una infusión de Prostaglandina E1. En este caso puede ser necesaria instaurar ventilación mecánica y soporte inotrópico. El concepto relevante a tener en mente en esta patología ductus dependiente es, que la perfusión sistémica (total o parcial) depende del ductus con shunt de derecha a izquierda a través de esta estructura.

En estos pacientes el cierre del ductus determina un aumento de la postcarga del ventrículo izquierdo, que de presentarse de manera aguda puede conducir a colapso cardiovascular, si es progresivo puede presentarse con disfunción ventricular izquierda con eventual isquemia subendocárdica. En este escenario puede desencadenarse isquemia mesentérica y producirse un cuadro de enterocolitis necrotizante, (ECN), que puede requerir cirugía (y convertirse en un paciente que puede requerir anestesia por un no especialista en anestesia cardiovascular). Los niños mayores, en los cuales la patología resulta inadvertida inicialmente o es menos severa, se presentan con un cuadro clínico en que se evidencia una diferencia en los pulsos y presiones de ambos hemicuerpos, aumento de la circulación colateral e hipertrofia del VI. (Figura 6. Coartación de la Aorta).

\section{Anestesia}

Un neonato con coartación aórtica que requiera una cirugía no cardiaca, será presumiblemente en el contexto de una urgencia. Es fundamental una evaluación adecuada de la patología, de las repercusiones sistémicas (falla cardiaca, acidosis metabólica e hiperlactacidemia), presencia de falla renal o respiratoria. En el período intra-operatorio es indispensable el monitoreo invasivo, tanto para el registro de la presión sistémica como para la toma seriada de exámenes de laboratorio (hematocrito, $\mathrm{pH}$, gases arteriales, lactato, pruebas de coagulación y recuento de plaquetas). Recordar que la instalación de la línea arterial sólo puede hacerse en la extremidad superior derecha y puede compararse con monitoreo no invasivo en una de las extremidades inferiores. Un acceso venoso

Médico Anestesiólogo, Hospital Luis Calvo Mackenna, Clínica Las Condes.

2 Médico Cirujano Cardiovascular Pediátrico, Hospital Luis Calvo Mackenna, Clínica Santa María.

Correspondencia:

Silvana Cavallieri Badilla

E-mail: silvanacavallieri@gmail.com

Conflicto de interés declarado: ninguno. 


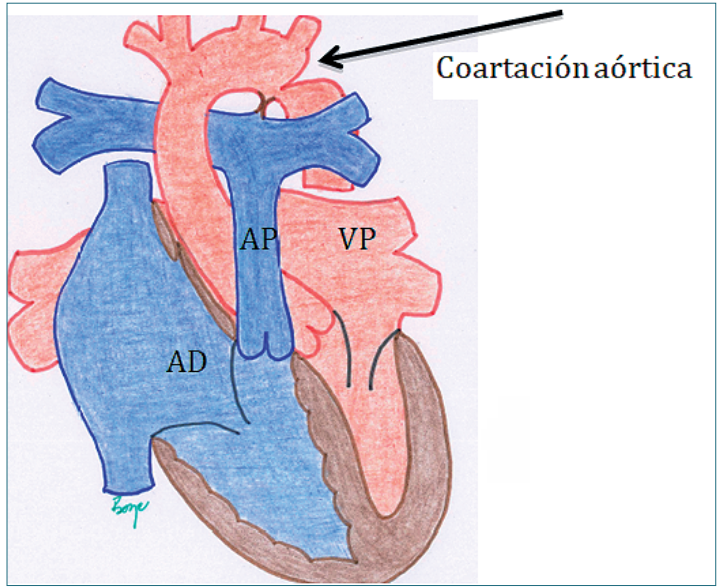

Figura 6. Coartación Aórtica: anomalía caracterizada por una estrechez distal a la emergencia de los vasos del cuello, en la unión del istmo aórtico y la aorta descendente.

central multi-lumen es mandatorio, sobre todo si se presume el uso de agentes vasoactivos. El monitoreo de la diuresis también debe realizarse. Los medicamentos anestésicos no deben deprimir ni la función cardiaca ni la resistencia sistémica. La resistencia vascular pulmonar debe mantenerse alta y no se debe hiperventilar ni administrar una fracción de oxígeno inspirada alta, aunque esto debe balancearse con una oxigenación adecuada del neonato. Se debe asegurar un hematocrito óptimo (alrededor $40 \%$ ) y un aporte cuidadoso de volumen en forma preferentemente de coloides.

En niños mayores, el síntoma principal es hipertensión en el territorio proximal a la estrechez, dependiendo de cuán acentuada sea la misma (Figura 6). Es relevante revisar los exámenes previos, particularmente la ecocardiografía, para tener noción de la magnitud de la coartación. Durante la anestesia debe evitarse disminuir la contractilidad para no disminuir la perfusión distal a la CoAo ni la perfusión coronaria. Tampoco exacerbar la hipertensión en el territorio sobre la coartación y deben evitarse los estímulos que agraven esta hipertensión. Dentro de los halogenados, todos son adecuados a concentraciones clínicas. El uso de anestesia regional neuroaxial en estos pacientes debe evitarse ya que el grado de circulación colateral en el nivel de la médula suele ser relevante y por lo tanto aumenta el riesgo de sangrado durante el procedimiento. (Tabla 8 y 9. Grilla de manejo del paciente neonato y paciente pediátrico portador de coartación aórtica).

Debe tenerse en cuenta que los pacientes con

\begin{tabular}{lc}
$\begin{array}{l}\text { Tabla 8. Grilla de manejo del paciente neonato } \\
\text { portador de coartación de la aorta }\end{array}$ \\
\begin{tabular}{lc} 
Contractilidad & $=$ \\
F Cardiaca & $=$ \\
Precarga & $\uparrow$ \\
RVS & \multicolumn{1}{c}{$\begin{array}{c}\text { ( o } \uparrow \text { (para mejorar flujo a } \\
\text { través del ductus al territo- } \\
\text { rio sistémico) }\end{array}$} \\
RVP & Mantener infusión de PGE \\
Mantener ductus permea- \\
ble
\end{tabular} \\
\hline
\end{tabular}

Tabla 9. Grilla de manejo del paciente pediátrico portador de coartación de la aorta

\begin{tabular}{lcc}
\hline Contractilidad & $=$ \\
F Cardiaca & $=$ \\
Precarga & $=$ \\
RVS & $\begin{array}{l}=\text { (no provocar hiperten- } \\
\text { sión) }\end{array}$ \\
RVP & & \\
\hline
\end{tabular}

coartación aortica reparada pueden tener estenosis residuales (verificar siempre la presión en las extremidades superiores e inferiores) y pueden también presentar hipertensión sistémica, algún grado de disfunción ventricular y enfermedad coronaria.

\section{Estenosis de la válvula aórtica}

La estenosis valvular aórtica (EA) es una lesión de la válvula que produce obstrucción del tracto de salida del ventrículo izquierdo, dificultando la eyección de éste por aumento de la postcarga. La consecuencia de este trastorno es la hipertrofia ventricular izquierda, que lleva a disfunción diastólica con aumento de la presión de fin de diástole. Sin embargo, la lesión puede producirse o asociarse a obstrucción en otros niveles, tanto en la región supra-valvular o sub-valvular, aunque estas dos últimas ubicaciones son menos frecuentes, (la obstrucción a nivel del anillo es lejos la más frecuente y alcanza el $70 \%$ de todos los casos).

La estenosis aórtica es una patología que puede formar parte del espectro del Síndrome de Hipoplasia del Corazón Izquierdo (SHCl) y estos pacientes caen en la categoría de ventrículo único, (tema que se revisará en una sección posterior en este artículo). La 
estenosis aórtica da cuenta de aproximadamente el $5 \%$ de todas las cardiopatías y hay predominancia en el sexo masculino. Se asocia también a algunas condiciones genéticas (Síndrome de Turner o 45XO) y también es más prevalente cuando existe consanguinidad.

Una forma frecuente de presentación es la Válvula Aórtica Bicúspide, (VAB), globalmente es la cardiopatía más frecuente de todas y puede afectar hasta el 1,5\% de los adultos, siendo muchas veces un hallazgo sin significancia patológica. Su forma de presentación más frecuente es por fusión de los velos coronarianos derecho e izquierdo (aproximadamente $80 \%$ ), luego viene por fusión de los velos coronariano derecho y no coronariano (15\% aproximadamente) y mucho menos frecuente es por fusión de los velos coronarianos izquierdo y no coronariano. La VAB verdadera también es rara. Si lo vemos desde otro punto de vista, la asociación de CoAo con VAB también es frecuente.

A pesar que la VAB puede presentarse como un hallazgo, es importante en cardiología saber cuál es el origen de esa $V A B$, ya que la $V A B$ por fusión de los velos derecho y no coronariano, es la que más frecuentemente se encuentra asociado a estenosis e insuficiencia aórtica y dilatación de la raíz y aorta ascendente. Esto sucede debido a que hay una alteración de las proteínas estructurales de la pared de estas estructuras, lo que condiciona una predisposición a presentar una disección aórtica. (Figura 7: Estenosis de la aorta).

\section{Fisiopatología}

Al existir una obstrucción a la eyección ventricular izquierda se produce una hipertrofia del ventrículo proporcional al grado de obstrucción. Si la obstrucción es severa provocará una hipertrofia severa y la posibilidad de isquemia miocárdica debido a la combinación de débito cardiaco disminuido, aumento del consumo de oxígeno miocárdico por la mayor masa ventricular y a disminución de la presión de perfusión por aumento de la presión final de diástole del VI.

Si esta obstrucción es temprana y severa en el período fetal podría resultar en un menor desarrollo de las cavidades izquierdas llegando hasta el $\mathrm{SHCl}$. Se pueden distinguir, a grosso modo, dos cuadros distintos de presentación para la estenosis aórtica. El primero se presenta en los recién nacidos que presentan estenosis aórtica crítica y se encuentran en una situación muy desfavorable ya que podrían ser dependientes del ductus arterioso para asegura el débito

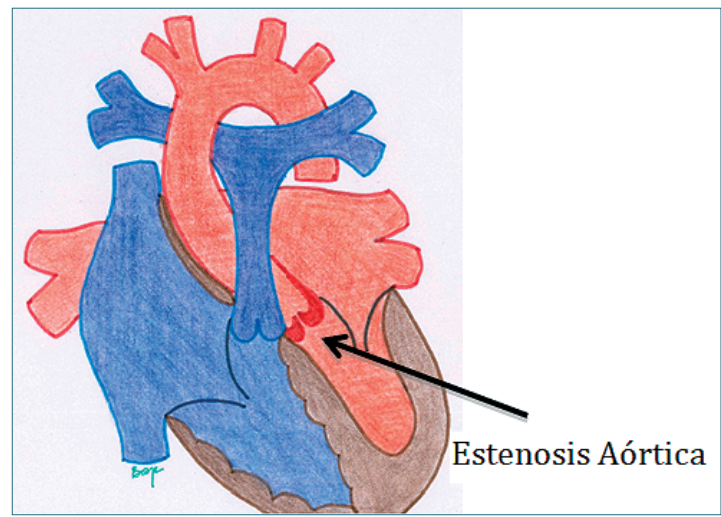

Figura 7. Estenosis de la Aorta. Esta patología pertenece al grupo de lesiones que provocan Obstrucción del Tracto de Salida del Ventrículo Izquierdo (OTSVI). Ésta causa una dificultad en la eyección de sangre a la aorta desde el ventrículo izquierdo, resultando en hipertrofia y disfunción diastólica del ventrículo izquierdo y aumento de la presión retrógrada de esas cavidades (atrio izquierdo, venas pulmonares, arterias pulmonares y finalmente ventrículo derecho).

sistémico (patología ductus dependiente por lo tanto se encontrará bajo tratamiento con PGE1). Cuando el ductus se cierra el paciente cae en un hipo débito severo con compromiso de la circulación sistémica, con pronóstico ominoso si no se trata rápidamente. Estos recién nacidos raramente llegaran a requerir anestesia para una cirugía no cardiaca excepto que presenten una patología como ECN. La segunda forma de presentación es una estenosis aórtica no crítica. Los neonatos y niños con estenosis aórtica no crítica pueden manifestar retardo en el crecimiento y síntomas de congestión pulmonar. En todo caso, si no hay una clara hipoplasia de las cavidades izquierdas que se manifiestan en el período neonatal, la estenosis aórtica puede ser asintomática y descubrirse por la presencia de un soplo sistólico precordial en el foco aórtico. En otros casos cuando la obstrucción es severa puede existir dolor precordial o síncopes en relación con el ejercicio.

El estándar de oro para definir el grado de obstrucción es la cateterización cardiaca, pero no es una información que esté disponible en la mayor parte de los pacientes, por lo que es la ecocardiografía transtorácica el examen que más frecuentemente se dispondrá en la evaluación pre anestésica, y nos señalará la gradiente, es decir pérdida de presión a través de la válvula aórtica en sístole. En todo caso el gradiente medido por la ecocardiografía muchas veces refleja el grado de obstrucción en reposo y no da cuenta de la obstrucción en condiciones de stress como llanto 
o ejercicio. En la Tabla 10 se entregan los valores de severidad de EA en niños.

\section{Anestesia}

Para la adecuada planificación de la anestesia es fundamental conocer el grado de obstrucción de la válvula aórtica, que se evalúa como ya se mencionó a través de la gradiente entre el ventrículo izquierdo y la aorta durante sístole. En el neonato con estenosis aórtica ductus dependiente es fundamental conservar el ductus permeable, por lo que debe mantenerse la infusión de PGE1 y se debe evitar el uso de depresores miocárdicos o anestésicos que disminuyan la RVS. El cierre del ductus en estos pacientes conlleva la aparición de shock cardiogénico severo. En el paciente lactante o niño con estenosis aórtica crítica igualmente se debe mantener la presión arterial sistémica, disminuir el consumo de oxígeno y evitar la disminución de la frecuencia cardiaca durante el período perioperatorio. En términos generales, fármacos como el propofol e isoflurano, que disminuyen marcadamente la RVS, están contraindicados, al menos en un primer momento, mientras se evalúa el comportamiento del paciente durante la anestesia.

Es importante mencionar que la EA supravalvular tiene un comportamiento algo distinto y más desfavorable en el contexto anestésico respecto a la EA valvular y subvalvular. Frecuentemente la EA supravalvular se presenta con estenosis de las ostias coronarias e incluso de sus trayectos iniciales. Esta condición de estenosis aórtica supravalvular es frecuente en los pacientes portadores de síndrome de Williams y se debe tener presente, ya que como en la cardiopatía coronaria, al inducir anestesia, vasodilatar el territorio arterial sistémico junto con el coronario en combinación a una estenosis fija entre la aorta y las coronarias perforantes, puede provocarse una isquemia coronaria que lleve al paro cardiaco en la inducción anestésica. Por esto es imperativo que no se vasodilate al paciente. La Ketamina puede ser un medicamento útil en la inducción para esta población. (Tabla 11 y 12: Grilla de manejo paciente neonato y pediátrico portador de estenosis aórtica).

\section{Transposición de grandes arterias}

La transposición de las grandes arterias (TGA) es una de las enfermedades cardiacas congénitas cianóticas más comunes. Se presenta entre el 5-7\% de todas las cardiopatías y es más frecuente en varones.

\begin{tabular}{|c|c|c|}
\hline Grado & $\begin{array}{c}\text { Gradiente Me- } \\
\text { dio } \\
(\mathrm{mmHg})\end{array}$ & $\begin{array}{c}\text { Área Valvular } \\
\text { Aortica } \\
\left(\mathrm{cm}^{2}\right)\end{array}$ \\
\hline Leve & $<25$ & $>1,5$ \\
\hline Moderado & $25-40$ & $1,0-1,5$ \\
\hline Severo & $>40$ & $<1,0$ \\
\hline Muy Severa & $>70$ & $<0,6$ \\
\hline
\end{tabular}

\section{Tabla 11. Grilla de manejo del paciente neona-} to portador de estenosis aortica crítica

\begin{tabular}{ll}
\hline Contractilidad & $=$ \\
F Cardiaca & $=$ \\
Precarga & $\uparrow$ \\
RVS & \multicolumn{1}{c}{$\begin{array}{c}1 \\
\text { RVP }\end{array}$} \\
& $\begin{array}{l}\text { través del ductus al territo- } \\
\text { rio sistémico) }\end{array}$ \\
Mantener ductus permea- & Mantener infusión de PGE \\
ble & \\
\hline
\end{tabular}

\section{Tabla 12. Grilla de manejo del paciente pediá-} trico portador de estenosis aortica

\begin{tabular}{lc}
\hline Contractilidad & $=$ \\
F Cardiaca & $=$ o ligera $\downarrow$ \\
Precarga & $=$ \\
RVS & $\uparrow$ (no permitir caída) \\
RVP & $=$ \\
\hline
\end{tabular}

En la TGA el defecto principal consiste en que la arteria aorta emerge desde el ventrículo derecho y la arteria pulmonar emerge desde el ventrículo izquierdo. Hay una situación anatómica en que hay concordancia atrio-ventricular pero discordancia ventrículo-arterial. Esto quiere decir que la atrio derecho se corresponde con el ventrículo derecho y la aurícula izquierda se corresponde con el ventrículo izquierdo pero ambos ventrículos dan nacimiento a la arteria que no les corresponde normalmente. Para que el paciente sobreviva debe existir una zona de mezcla de la sangre que consiste en una comunicación interatrial. 


\section{Fisiopatología}

Se trata de una patología en la que existe una circulación en paralelo en lugar de una circulación en serie como es lo normal. La sangre venosa sistémica (con $\mathrm{P}_{\mathrm{a}} \mathrm{O}_{2}$ baja) que vuelve al atrio derecho, pasa al ventrículo derecho, de ahí a la aorta y luego de perfundir el organismo vuelve por el retorno venoso sistémico nuevamente al atrio derecho. Por el otro lado, la sangre venosa pulmonar (con $\mathrm{P}_{\mathrm{a}} \mathrm{O}_{2}$ elevada) que vuelve al atrio izquierdo, pasa al ventrículo izquierdo, de ahí a la arteria pulmonar y luego de oxigenarse en los pulmones, retorna nuevamente al atrio izquierdo. Por lo tanto hay dos circuitos sanguíneos, ambos separados y en paralelo.

Para que el paciente sobreviva necesariamente debe existir un nivel de mezcla. Los síntomas del paciente dependerán, entre otros, de la existencia y cuán amplia sea la zona de mezcla. Cuando la mezcla no es lo suficientemente adecuada para satisfacer las demandas de oxígeno del organismo es necesario que el niño reciba PGE1 para evitar el cierre del ductus (una alternativa para aumentar el flujo pulmonar y con ello mejora la oxigenación debido a mezcla a nivel atrial). En ocasiones es necesario realizar una septostomía atrial con balón (septostomía percutánea de Rashkind) para ampliar la comunicación interatrial y asegurar el nivel de mezcla: desde el atrio izquierdo al atrio derecho. La cirugía correctora suele realizarse dentro de la primera semana de vida si las condiciones del paciente lo permiten, (si no hay infección asociada). En raras ocasiones el paciente puede presentar un cuadro de ECN que requerirá manejo médico u ocasionalmente quirúrgico. En otras ocasiones el paciente portador de una TGA puede presentar una malformación asociada que requiera solucionarse en forma urgente, y puede corresponderle a un anestesiólogo general hacerse cargo de un paciente de esta categoría. (Figura 8: Transposición de Grandes Arterias).

\section{Anestesia}

En la evaluación es relevante saber que grado de mezcla tiene el paciente revisando las condiciones clínicas y la ecocardiografía más reciente. Registrar cual es la saturación de oxígeno actual $\left(\mathrm{S}_{\mathrm{a}} \mathrm{O}_{2}\right)$, la concentración de hemoglobina (manejar la anemia si existiere) y el estado ácido-base. Si hay acidosis e hiperlactacidemia realizar una reanimación con fluidos, preferentemente albúmina, drogas vasoactivas y eventualmente usar bicarbonato a razón de $1 \mathrm{mEq} / \mathrm{Kg}$ en bolos lentos. Durante el período perioperatorio el objetivo es man-

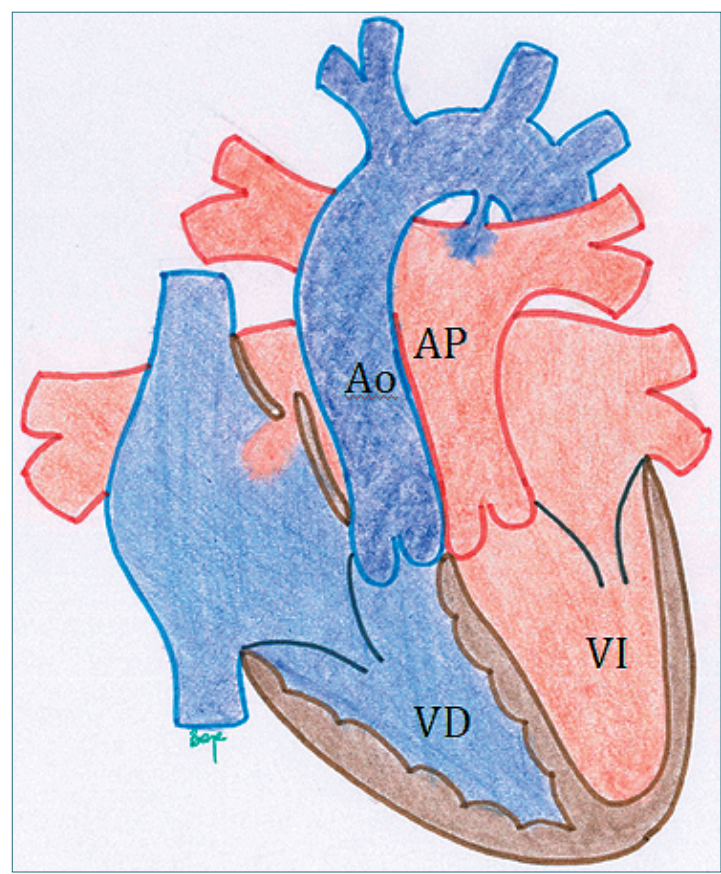

Figura 8. Transposición de las Grandes Arterias: Patología clásica de la circulación en paralelo. Ambos circuitos circulatorios están separados, el sistémico impulsado por el ventrículo derecho y el pulmonar, por el ventrículo izquierdo. El resultado es que la circulación sistémica hace circular sangre desaturada y el pulmonar sangre saturada. Para mantener al recién nacido vivo, se necesitan niveles de mezcla sanguínea, esto ocurre a través del ductus arterioso que aumenta el flujo pulmonar y finalmente la mezcla de sangre oxigenada se produce a nivel atrial.

tener el flujo y la resistencia sistémica, evitando uso de depresores miocárdicos y favorecer la mezcla a nivel atrial (evitando la acidosis e hipercapnia). En la medida que se mejore la mezcla atrial, más sangre oxigenada llegara al atrio derecho y mejor será la saturación de oxígeno de la mezcla que se distribuirá al territorio sistémico, a través de la arteria aorta. (Tabla 13: Grilla de manejo del paciente neonato portador de TGA).

\section{Ventrículo único}

Es un término que engloba varios y diferentes defectos anatómicos cardiacos o tipos de circulación presentes al nacimiento que comparten el mismo problema: el sistema circulatorio pulmonar y sistémico están en paralelo, sumado a mezcla sanguínea intracardiaca total. Por definición son cardiopatías cianóticas, debido a la mezcla total intracardiaca de ambos 


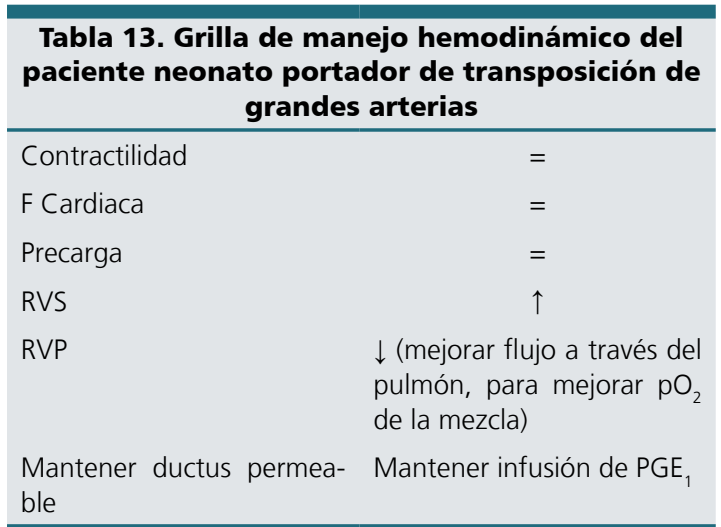

retornos venosos. A pesar de esto, frecuentemente el Qp:Qs está aumentado.

Del punto de vista anatómico, el Ventrículo Único (VU) incluye diversas malformaciones cardíacas que se presentan con una cavidad hipoplásica o un septum interventricular rudimentario, en las que resulta imposible o no es aconsejable reconstruir un corazón con dos ventrículos funcionales, (es decir no es posible tener circulación pulmonar y sistémica en serie). La historia natural es la muerte a edad precoz.

El interés para el anestesiólogo pediátrico general radica en que cada vez hay más niños en nuestro país con esta condición, ya sea en transición de la etapa I a la etapa II o de la etapa II a la etapa III o ya completada la etapa III. Para ello el anestesiólogo general debe tener un conocimiento básico de la fisiología de cada etapa para acomodar el manejo anestésico a lo que sea más conveniente para el paciente desde el punto de vista hemodinámico.

Patologías que se consideran Ventrículo Único: 1. Hipoplasia de Ventrículo Derecho (Ventrículo Único de Anatomía Izquierda)

a. Atresia Tricuspídea: esta patología puede presentarse en distintas variantes dependiendo de la relación de los grandes vasos, tamaño de la CIV (o foramen Bulbo Ventricular) y tamaño del anillo pulmonar. En cualquier caso el VD no recibe oferta de flujo sanguíneo, por lo que no se desarrolla. El destino es un ventrículo único de morfología izquierda.

b. Atresia Pulmonar con Septum Intacto (APSI). Existe un VD pequeño pero que no es posible reclutar y el flujo sanguíneo debe seguir la vía univentricular.

2. Hipoplasia de Ventrículo Izquierdo (Ventrículo Único de anatomía Derecha) a. Atresia Mitral: EI VI no se desarrolla por falta de flujo a través de la válvula mitral. Habitualmente se presenta con CIV y variabilidad en la posición de la salida aórtica. El desarrollo de la aorta también se compromete.

3. Síndrome de Hipoplasia del Corazón Izquierdo. Las estructuras del lado izquierdo son hipoplásicas, desde el anillo mitral hasta la zona ductal. Su etiología sería la desviación a izquierda del septum primum interatrial.

4. Ventrículo Único de Doble Entrada: son combinaciones de lesiones a múltiples niveles cardiacos, sin embargo a la cavidad ventricular se abren ambas válvulas AV (con grado variable de hipoplasia de una $u$ otra).

5. Ventrículo Único de Doble Salida: Combinación de lesiones a distintos niveles cardiacos, el resultado es que sobre esta cavidad ventricular funcionalmente única, emergen ambas válvulas semilunares (con grado variable de hipoplasia de una u otra).

Las siguientes ilustraciones dan cuenta de dos de las patologías que entran en el grupo considerado ventrículo único. (Figuras 9 y 10).

A consecuencia de la anomalía estructural cardiaca, se produce una mezcla completa del retorno venoso sistémico y pulmonar a nivel atrial y/o ventricular, existiendo un solo ventrículo funcional que distribuye el gasto cardíaco tanto al lecho vascular pulmonar como sistémico (circulación en paralelo). La eyección ventricular es la suma del flujo sanguíneo pulmonar (Qp) y sistémico (Qs) y la distribución del flujo sanguíneo hacia los territorios pulmonar y sistémico depende de las resistencias relativas de ambos territorios.

Las variables que determinarán el Qp:Qs serán:

- La $\mathrm{S}_{a} \mathrm{O}_{2}$, que es igual en la aorta y en la arteria pulmonar dado que la circulación es en paralelo con mezcla total.

- La saturación venosa sistémica.

- La saturación venosa pulmonar (que habitualmente se asume como 100\%).

Con estas variables podemos estimar la razón del flujo sanguíneo pulmonar y sistémico (Qp:Qs). La forma de calcular el Qp:Qs es mediante la relación de gastos cardiacos sistémico y pulmonar, datos que solamente pueden ser obtenidos con estudio hemodinámico. Afortunadamente está disponible una forma simplificada para obtener este dato: 


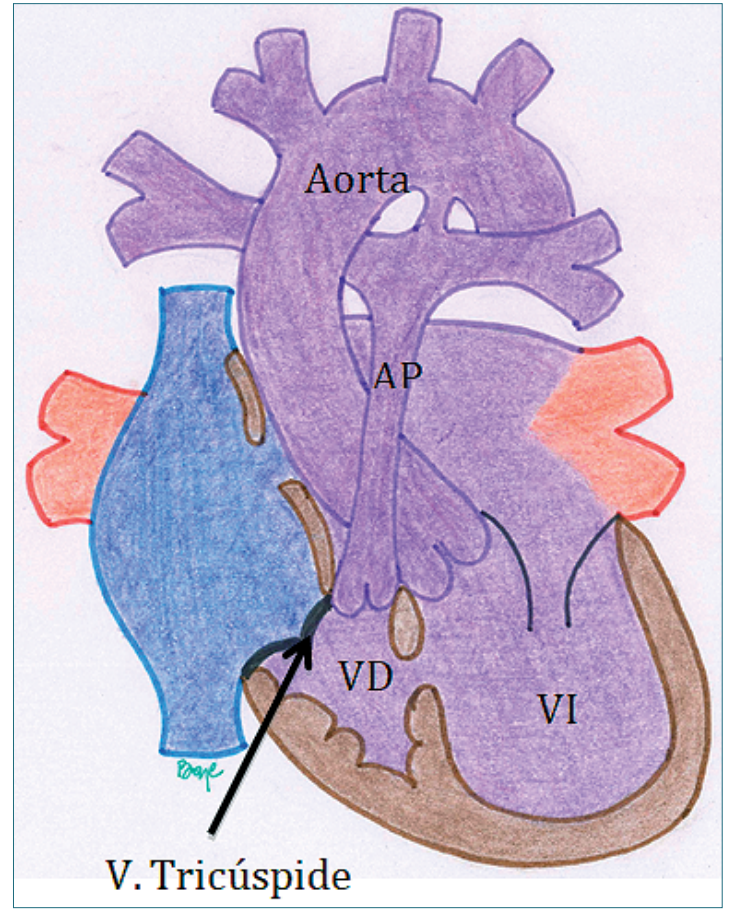

Figura 9. Ventrículo Único tipo Atresia Tricuspídea. En esta patología, hay una ausencia del desarrollo de la válvula tricúspide, con la consecuencia de la hipoplasia del ventrículo derecho. El retorno venoso sistémico debe avanzar a través del septum interatrial hacia el atrio izquierdo, lugar de mezcla con la sangre saturada del retorno venoso pulmonar. Ésta pasa al ventrículo izquierdo impulsando la sangre hacia la circulación sistémica. El flujo pulmonar es aportado tanto por el ductus arterioso persistente (DAP) como por el flujo anterógrado que pasa por la comunicación interventricular y la válvula pulmonar. Esta patología solamente puede seguir la vía univentricular hasta completar la unión cavopulmonar total (procedimiento de Fontan).

$$
\frac{Q_{p}}{Q_{S}}=\frac{S_{A o} O_{2}-S_{V M} O_{2}}{S_{V P} O_{2}-S_{A P} O_{2}}
$$

La fórmula toma las saturaciones de la aorta (Ao), Arteria Pulmonar (AP), venosa central o mixta (VM) y venas pulmonares (VP). Con la oximetría de pulso se obtienen los valores de Ao y AP y se asume que la saturación de las venas pulmonares es $100 \%$, por lo que el único dato que faltaría obtener es la saturación central, la que se sugiere obtener de la vena cava superior, esto porque un catéter venoso central

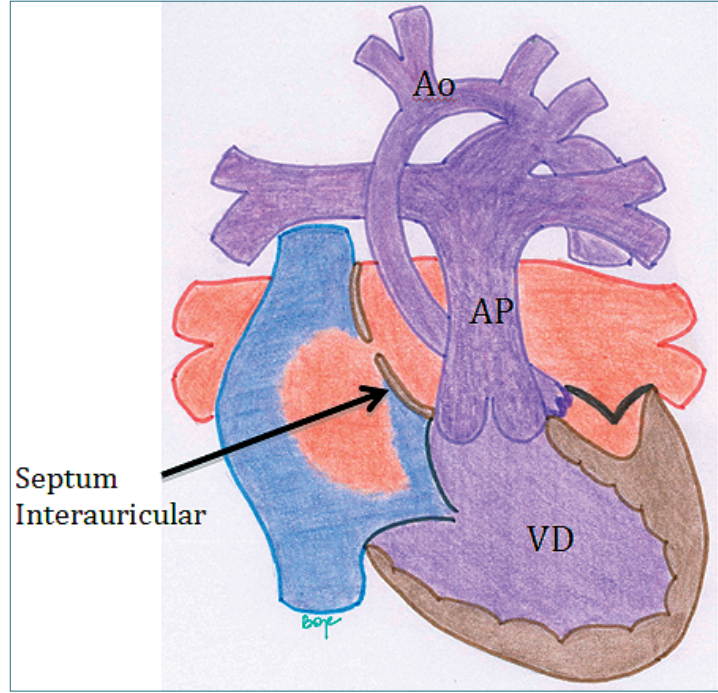

Figura 10. Ventrículo Único tipo Síndrome de Hipoplasia de Corazón Izquierdo (SHCl) subtipo Atresia Mitro-Aórtica (AMAA). En esta patología, hay una ausencia del desarrollo de la válvula mitral, con la consecuente hipoplasia del ventrículo izquierdo y las estructuras sobre él (aorta ascendente y arco aórtico). El retorno venoso pulmonar debe avanzar a través del septum interatrial hacia el atrio derecho, lugar de mezcla con la sangre desaturada del retorno venoso sistémico. Ésta pasa al ventrículo derecho el que impulsa la sangre hacia la circulación sistémica, a través de la arteria pulmonar. El flujo sistémico es asegurado por el ductus arterioso persistente (DAP), el que distribuye la sangre hacia el hemicuerpo inferior y de manera retrógrada por el arco aórtico en dirección de las arterias coronarias (la aorta ascendente severamente hipoplásica funciona como arteria coronaira unica). El flujo pulmonar es otorgado por la emergencia de las ramas pulmonares desde la arteria pulmonar. A pesar de que es una patología cianótica, dado por la mezcla total de sangre en el coazón, habitualmente el flujo pulmonar está severamente aumentado. Esta patología solamente puede seguir la vía univentricular etapificada inicialmente con el procedimiento de Norwood, hasta completar la unión cavopulmonar total (procedimiento de Fontan).

cuya punta esté a nivel atrial, entregará información errónea de la $\mathrm{S}_{V M} \mathrm{O}_{2}$ debido a que estará falsamente elevada por el aporte de las venas pulmonares.

Entonces, es necesario entender que los niños portadores de un ventrículo único deben ser sometidos a varias cirugías (e intervenciones hemodinámicas) a lo largo de su vida para poder preparar al corazón y la vasculatura pulmonar para lograr un circuito en serie, a expensas de que el retorno venoso sistémico avance de manera pasiva (sin que exista un ventrículo que la impulse) por el pulmón hasta el atrio sistémico. Esto 
es lo que se llama "Etapificación Univentricular". El pronóstico de los pacientes que van hacia la vía univentricular ha mejorado notablemente en las últimas décadas. Los niños que son manejados cuidadosamente en su infancia, (para así optimizar su anatomía y fisiología), pueden ser sometidos a la operación de Fontan (unión cavo-pulmonar total), con buen pronóstico y calidad de vida, con escasas restricciones e incluso, en el caso de las mujeres, de llegar a buen término un embarazo.

La Etapificación Univentricular ("staging") incluye tres pasos o etapas.

\section{Etapa I:}

El objetivo en esta etapa es el de asegurar el flujo sistémico sin obstrucción (tracto de salida del VU sin obstrucción) y regulación del flujo pulmonar. Para tales objetivos el paciente puede ser sometido a uno o varios procedimientos en la etapa neonatal:

1. Regulación del flujo pulmonar: El objetivo es lograr manejar Qp:Qs idealmente cercano a 1:1. Para esto se debe dejar solamente una fuente de flujo pulmonar:

- Aumentar el flujo pulmonar: Shunt Blalock-Taussig modificado, interponiendo una prótesis vascular entre el tronco braquiocefálico y la arteria pulmonar derechos. Asociado a cierre de DAP.

- Disminuir el flujo pulmonar: Banding de la arteria pulmonar. Asociado a cierre de DAP.

2. Asegurar flujo sistémico sin obstrucción: según la anatomía y la presencia de CoAo, a veces es necesario reparar el arco de la aorta mediante el Procedimiento de Norwood.

La Figura 11 ilustra el camino que puede seguir un recién nacido con $\mathrm{VU}$ funcional y/o anatómico.

La Figura 12 muestra los objetivos en el manejo del corazón univentricular en el periodo de recién nacido. El objetivo es equilibrar el flujo sistémico con el flujo pulmonar. El paciente necesariamente es cianótico. Con un Qp:Qs equilibrado la saturación óptima es entre $75 \%$ y $85 \%$. Si es mayor que $85 \%$ habla de un exceso de flujo pulmonar (hiperflujo), en desmedro del flujo sistémico lo que determina potencialmente hipodébito sistémico. Si es menor de $70 \%$, se habla de un flujo pulmonar disminuido.

La Figura 13 muestra la situación de un Síndrome de Hipoplasia de Corazón Izquierdo (SHCI) con reparación del arco aórtico tipo Norwood. En esta etapa se lograron los objetivos enunciados en el párrafo anterior:

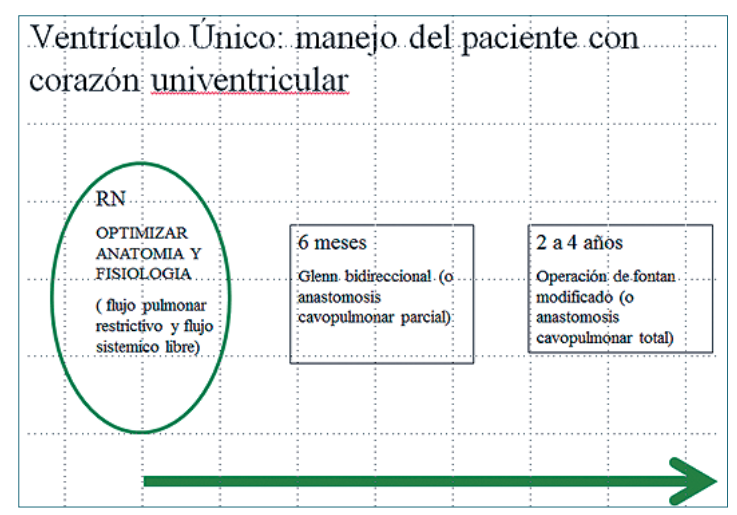

Figura 11. Etapas en el manejo del corazón Univentricular.

1. Se asegura un flujo sistémico no restrictivo mediante la reparación del arco de la aorta con la técnica de Norwood. Se elimina tanto el DAP (como tracto de salida sistémico, dependiente de PGE1), como la zona de CoAo.

2. Se asegura un flujo pulmonar conocido. Esto se hace a través de 2 opciones:

a. Conducto VD-AP o conducto de Sano: ésta es la fuente de flujo pulmonar más elegida actualmente. Permite más estabilidad postoperatoria con mejor sobrevida. El flujo pulmonar es sistólico y es enviado por el VD.

b. Shunt Sistémico-Pulmonar (Shunt Blalock-Taussig Modificado -SBT-). Esta fuente de flujo pulmonar se realiza a través de una prótesis vascular interpuesta entre el tronco arterial braquiocefálico y las ramas pulmonares, habitualmente a derecha. Es de postoperatorio más dificultoso ya que el flujo pulmonar es otorgado a través de esta fístula quirúrgica durante todo el ciclo cardiaco (sistodiastólico).

3. Se asegura la mezcla intracardiaca del retorno venoso sistémico y pulmonar a nivel atrial, mediante una CIA amplia.

\section{Anestesia para el paciente portador VU en etapa I}

Como se expuso en los párrafos previos, los pacientes tienen sobrecarga de volumen y de presión. Un solo ventrículo asume el débito sistémico y pulmonar al mismo tiempo. La condición hemodinámica más apropiada es mantener el Qp: Qs en alrededor de 1:1 para no sobrecargar el ventrículo.

El manejo anestésico debe estar destinado a man- 


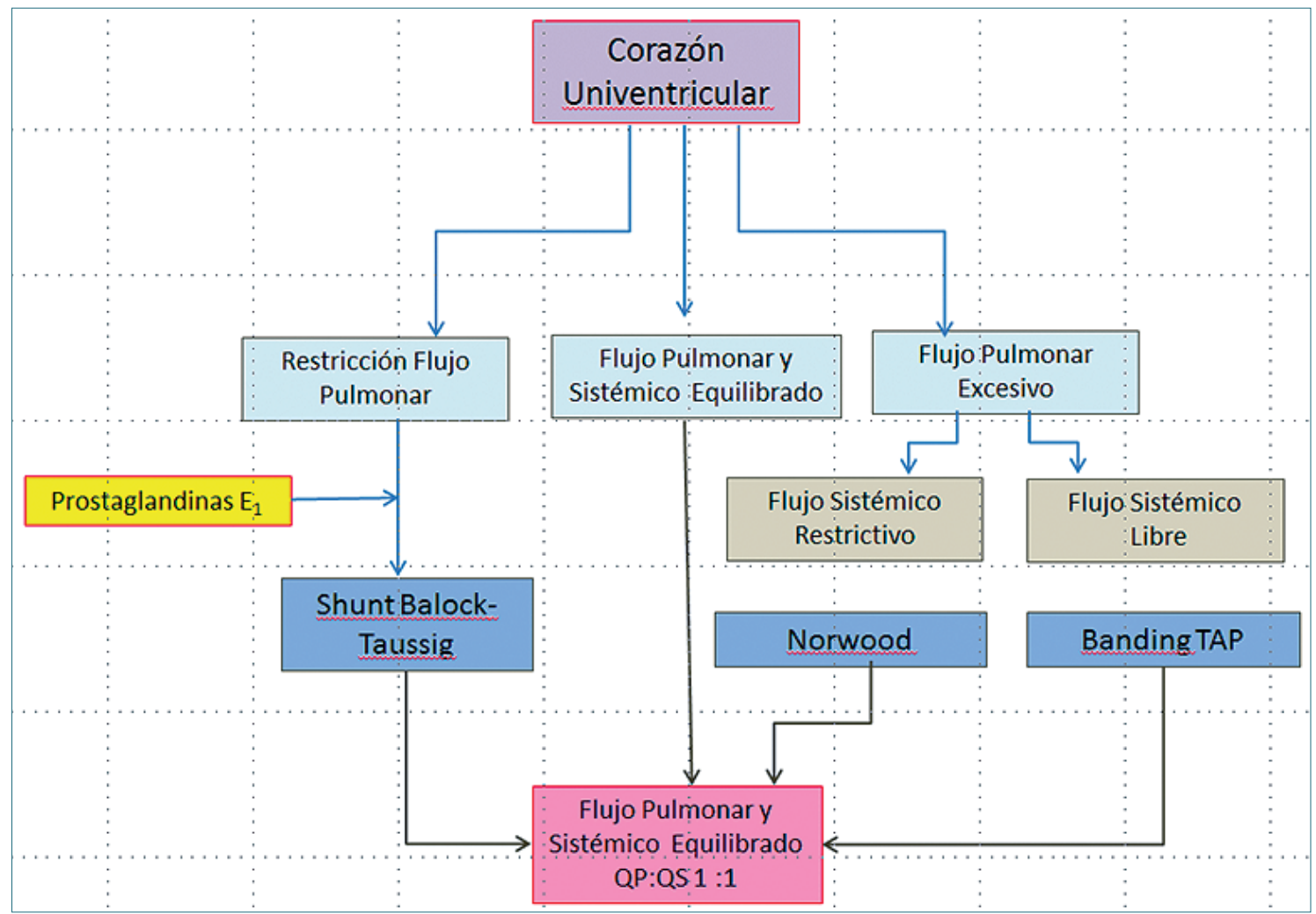

Figura 12. Manejo del corazón univentricular en el período de recién nacido.

tener la contractilidad y la relación entre ambos flujos. Los pacientes son cianóticos por la mezcla total. El hematocrito debe mantenerse sobre $40-45 \%$, la saturación en alrededor de 75-85\%, no mayor que eso (estimar que $\mathrm{S}_{\mathrm{a}} \mathrm{O}_{2}$ sobre $90 \%$ provoca Qp:Qs sobre 4:1). Un flujo pulmonar excesivo aumentará la saturación a expensas de disminuir el flujo sistémico, lo cual es contraproducente y deletéreo.

Entre los medicamentos anestésicos debe preferirse aquellos que no depriman la contractilidad ni disminuyan la resistencia sistémica. Para procedimientos dolorosos de corta duración como la instalación de un acceso venoso central una sedación con Ketamina puede ser apropiada siempre que haya una persona con experiencia vigilando la ventilación. Para otros procedimientos no dolorosos o poco dolorosos, como imágenes o endoscopias, la inducción y mantención con sevoflurano es apropiada, lo mismo si se trata de cirugía mayor: una inducción cuidadosa y mantención con sevoflurano. De utilizarse propofol, debe ser en dosis bajas y titulando muy cuidadosamente el efecto. Respecto al aporte de volumen, los pacientes no toleran la hipovolemia (el ayuno debe ser lo más limitado posible), pero tampoco se les debe sobrecargar de volumen. Es necesario tener en cuenta que los estudios de paro cardiorrespiratorio en niños mencionan la etapa I de un paciente portador de VU como una condición de particular riesgo de eventos hemodinámicos adversos durante la anestesia. Para una cirugía mayor o de emergencia se debe instalar monitoreo invasivo, tener un control frecuente del estado ácido base, mantener el hematocrito en niveles apropiados y eventualmente usar en forma profiláctica drogas vasoactivas como la dopamina. Es relevante tener una conducta preventiva de problemas más que intentar corregirlos una vez producidos los trastornos. Es muy importante saber de antemano que el tener una reanimación exitosa en el contexto de un PCR en pacientes en esta etapa es muy improbable, debido a la alta labilidad y escasa o nula reserva cardiaca. Una vez que el paciente entra en paro circulatorio, lo más probable es que no salga de ese estado.

El manejo postoperatorio debe realizarse siempre en una unidad de cuidados especializados. (Tabla 14. Grilla de manejo hemodinámico del paciente portador de VU en etapa I). 


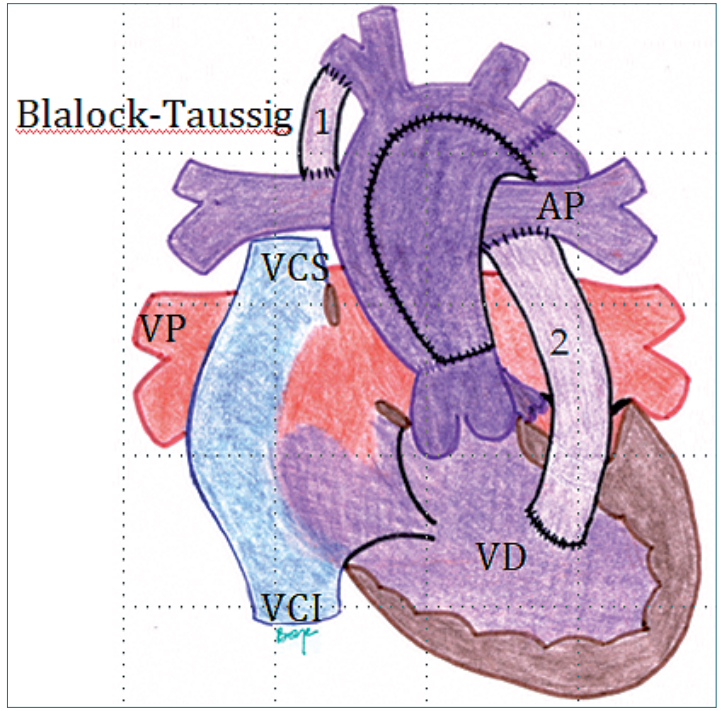

Figura 13. Ventrículo Único tipo $\mathrm{SHCl}$ con reparación del arco aórtico tipo Norwood. En esta etapa se busca asegurar un tracto de salida del ventrículo único sin obstrucción (mediante resección de tejido ductal y coartación de la aorta), mezcla intracardiaca de los retornos venosos sin restricción (mediante ampliación de CIA) y flujo pulmonar no dependiente de PGE1 (mediante un SBT [1] o conducto VD-AP [2]). Las fuentes de flujo pulmonar difieren una de otra principalmente en el flujo continuo (sisto-diastólico) del SBT respecto al flujo sistólico del conducto VD-AP.

\section{Etapa II: Operación de Glenn Bidireccional}

El procedimiento de Glenn Bidireccional (GBD) habitualmente se lleva a cabo alrededor de los 6 meses y consiste en:

1. Desconexión de VCS a la AD y anastomosis de ésta con las ramas pulmonares, dejando a la VCS como fuente de flujo para ambos pulmones.

2. Eliminar la comunicación entre el territorio venoso de la vena cava superior e inferior (ligadura de la vena Azygos).

3. Eliminar cualquier otra fuente de flujo pulmonar (como SBT, conducto VU-AP, colaterales sistémico-pulmonares o flujo anterógrado a través de la válvula pulmonar).

\section{De esta manera:}

a. Se elimina la sobrecarga de volumen y de presión del VU y del territorio pulmonar.

b. Se eliminan las comunicaciones venosas entre el hemicuerpo superior e inferior. El mantener estas comunicaciones hace que la sangre, al estar sometido a PVC elevadas, escape del territorio pulmonar al territorio venoso de la $\mathrm{VCl}$, provocando

\begin{tabular}{|c|c|}
\hline Contractilidad & Mantener \\
\hline F Cardiaca & Mantener \\
\hline Precarga & Mantener \\
\hline RVS & Mantener \\
\hline RVP & Mantener \\
\hline $\mathrm{FiO}_{2}$ & $\begin{array}{l}\text { Lo más baja posible para } \\
\text { mantener } \mathrm{SaO}_{2} 80-85 \%\end{array}$ \\
\hline
\end{tabular}

aún más desaturación.

c. Se otorga como fuente exclusiva de flujo pulmonar a la VCS (retorno venoso del hemicuerpo superior). Es importante recordar que la oferta de flujo pulmonar va a estar determinada por la cantidad de flujo cerebral. Si se hiperventila al paciente por lograr gasometría normales $\left(\mathrm{pCO}_{2}\right.$ entre 30 y $35 \mathrm{mmHg}$ ), lo que se va a lograr es que haya vasoconstricción de las arterias carótidas, esto hará que:

- Disminuya el flujo cerebral:

- Disminuya el flujo a ese territorio.

- Por derivación del flujo arterial sistémico hacia el hemicuerpo inferior, habrá aumento del retorno venoso desde la $\mathrm{VCl}$, territorio que no pasa a oxigenarse por los pulmones.

- Secundariamente se produce disminución del flujo pulmonar.

- El resultado es hipoxia progresiva, que no responde a aumento de la $\mathrm{FiO}_{2}$.

Es tentador elevar todos los parámetros del ventilador mecánico en este contexto, pero el resultado va a ser perpetuar el barrido de $\mathrm{CO}_{2}$ (aumentando la vasoconstricción (arotídea) y aumentar las presiones de ventilación, dificultando aún más el paso de sangre por el pulmón.

La recomendación es conocer la fisiología de esta etapa, la fuente anatómica del flujo pulmonar y que este circula a través de los pulmones de manera pasiva. Sólo así se puede anestesiar en forma segura a un paciente en etapa de Glenn.

El VU en etapa I, incluso en condición hemodinámica óptima, tiene algo de sobrecarga de volumen del VU (considerado como shunt de izquierda a derecha). La etapa II logra eliminar las fuentes de esa sobrecarga. En la década de los 90's se comenzó a aplicar el GBD como etapa intermedia al Fontan, lográndose reducir la mortalidad. Esto se hace muy evidente en el manejo de la hipoplasia del corazón 
izquierdo. Dado que en los niños pequeños la vena cava superior significa alrededor de 2/3 del retorno venoso sistémico, al realizarse la anastomosis de la VCS con la APD, se logra un flujo pulmonar adecuado para las necesidades del paciente. Las desventajas son que PVC esta elevada en el territorio de la VCS (puede incluso haber un síndrome de vena cava superior con edema en esclavina y cianosis marcada) (Figura14).

Es importante destacar que a medida que el paciente crece, va incrementando su nivel de cianosis, las causas son:

a. Crecimiento somático: a medida que el niño crece el hemicuerpo superior se va haciendo proporcionalmente menor que el hemicuerpo inferior, lo que conlleva disminución progresiva y proporcional del flujo pulmonar que es proporcionado por la VCS. Cabe recordar que en el adulto el flujo de la VCS llega a ser algo así como 1/3 del retorno sistémico. La representación del territorio de la $\mathrm{VCl}$ toma mayor importancia.

b. Fístulas Arterio-Venosas intrapulmonares: el dejar a la VCS como única fuente de flujo pulmonar y eliminar las fuentes extra de flujo sanguíneo al pulmón, deja la circulación pulmonar sin el aporte de un factor hepático (hasta el día de hoy no identificado), que sería inhibidor de la angiogénesis. El resultado es el desarrollo de vasos que comunican el territorio pulmonar arterial precapilar con el territorio venoso postcapilar, que no pasa por las unidades alveolares, sin que la sangre se oxigene.

c. Comunicaciones veno-venosas entre el territorio de las venas cavas superior e inferior.

\section{Manejo anestésico del VU en etapa II (GBD)}

Hay una singularidad en estos pacientes que conviene tener en mente y es que la hipercapnia (45 a 55 $\mathrm{mmHg}$ ) en pacientes con fisiología GBD, produce una mejoría de la $\mathrm{pO}_{2}$. Esto sucede por un aumento en el flujo sanguíneo cerebral motivado por la hipercarbia, lo que provoca un incremento del retorno venoso de la cava superior que finalmente optimiza el flujo pulmonar y la oxemia. Este efecto supera la vasoconstricción pulmonar causada por la hipercarbia. La utilidad de la maniobra es limitada, es decir puede permitirse un nivel de hipercarbia. moderada, pero si aumenta demasiado la $\mathrm{P}_{\mathrm{a}} \mathrm{CO}_{2}$, no hay mayor aumento de la $\mathrm{P}_{\mathrm{a}} \mathrm{O}_{2}$.

Tampoco es apropiado un aumento excesivo de la resistencia pulmonar, ya que el flujo de la VCS es pasivo a través de los pulmones. Por otro lado, el hiperventilar a estos pacientes en la inducción anestésica, puede provocar un barrido del $\mathrm{CO}_{2}$ que resulte en una

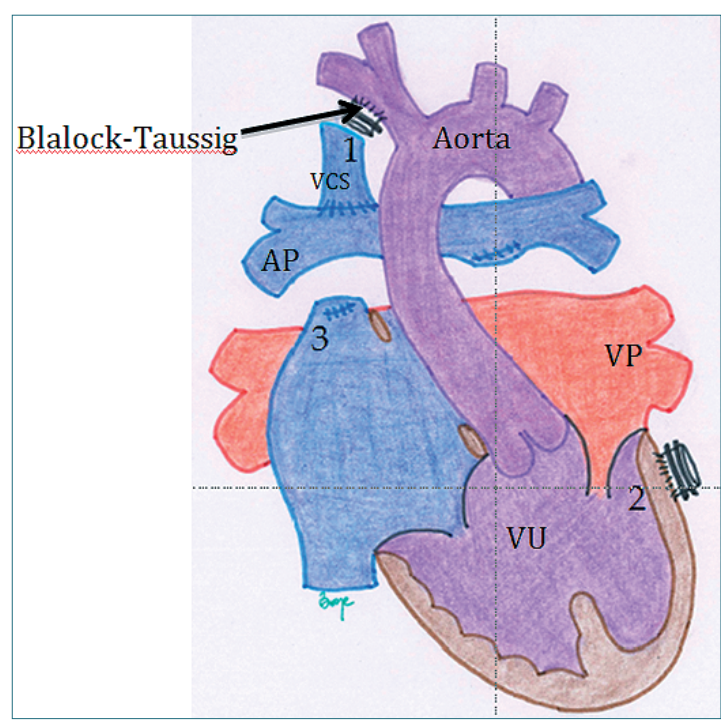

Figura 14. Ventrículo Único. Etapa de Glenn o Unión CavoPulmonar Superior. Se comienzan a separar los retornos venosos sistémico y pulmonar quedando conectada la VCS directamente a la AP. Al mismo tiempo se eliminan todas las fuentes de flujo pulmonar tales como el SBT (1) o el conducto VU-AP (2) que se habían realizado en la etapa I, las colaterales sistémico-pulmonares significativas y se elimina el flujo anterógrado por la válvula pulmonar seccionando y ligando esta estructura (3). También se elimina la comunicación entre el territorio venoso sistémico superior e inferior (es decir se liga la vena azygos).

vasoconstricción carotídea, que finalmente va a disminuir el aporte sanguíneo pulmonar. Un paciente en esta condición puede tolerar una cirugía laparoscopia, con insuflación de $\mathrm{CO}_{2}$, manteniendo en todo caso una presión intra-abdominal en límites razonables a la edad pediátrica y a la condición del paciente.

Cualquier tipo de inducción y mantención es apropiada siempre y cuando sea cuidadosa y titulando el efecto. Es apropiado resguardar el volumen intravascular ya que estos pacientes no toleran la hipovolemia. Debe evitarse cualquier maniobra que aumente la RVS. Dado que necesariamente son pacientes cianóticos el hematocrito debe estar alrededor de 40 a 45\%, ya que es la única manera de mantener un adecuado transporte de oxígeno. (Tabla 15. Grilla para el manejo hemodinámico del paciente portador de Glenn).

\section{Etapa III : Operación de Fontan.}

En esta etapa se completa la derivación de la sangre venosa hacia el pulmón en forma directa (unión cavo-pulmonar total). El ventrículo queda sólo eyec- 


\begin{tabular}{ll}
$\begin{array}{l}\text { Tabla 15. Grilla para el manejo hemodinámico } \\
\text { del paciente portador de un Glenn }\end{array}$ \\
\hline Contractilidad & Mantener \\
F Cardiaca & Mantener (ritmo sinusal \\
ideal) & Elevada \\
Precarga & Mantener \\
RVS & $=$ pero permitir aumento \\
RVP & discreto CO ${ }_{2}^{\prime}$ para aumen- \\
& tar flujo cerebral. No hiper- \\
& ventilar \\
& No es relevante \\
$\mathrm{FiO}_{2}$ & \\
\hline
\end{tabular}

tando hacia el territorio sistémico y la sangre venosa pasa desde ambas cavas a las arterias pulmonares directamente a los pulmones, sin que haya una bomba impulsora como lo es el VD en una anatomía normal. El principio de la operación de Fontan está en que el ventrículo derecho no es indispensable para sobrevivir ${ }^{9}$, siempre y cuando exista un ventrículo sistémico con función apropiada.

Hay dos formas de conectar la $\mathrm{VCl}$ al territorio pulmonar:

1. A través de una prótesis vascular, desconectando la VCI del atrio. Esto crea un conducto extracardiaco, (Figura 15), por interposición de un tubo entre las ramas pulmonares y la $\mathrm{VCl}$. Esta es la técnica más frecuentemente utilizada en nuestro país.

2. A través de aislamiento y redirección de la sangre de la $\mathrm{VCl}$ por dentro del atrio hacia las ramas pulmonares, quedando un conducto con media pared de atrio y media pared de prótesis vascular. Esto es el Fontan tipo túnel lateral.

Para que esta intervención tenga éxito, deben cumplirse algunas premisas: la anatomía, resistencia y presión en el árbol pulmonar deben ser bajas, la(s) válvula(s) atrioventricular(s) debe(n) ser competente(s), debe asegurarse de una mezcla total intracardiaca (CIA amplia), no debe existir obstrucción en el tracto de salida del VU y la función ventricular debe ser adecuada.

Esta circulación lleva a una elevación de la PVC, la magnitud de ésta depende de la resistencia vascular pulmonar y de la función del ventrículo único (diastólica y sistólica), al cual drenan las venas pulmonares. Una de las formas de mitigar esta elevación de la PVC es crear una comunicación entre el tubo o tunel del Fontan y el atrio sistémico, llamada fenestración. Ésta

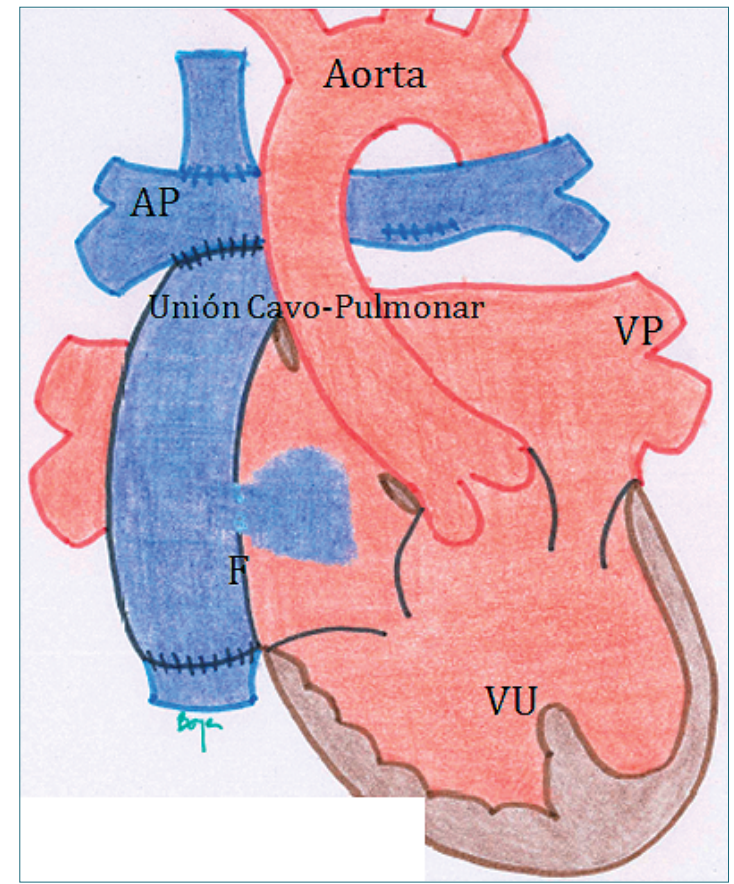

Figura 15. Ventrículo Único: Etapa de Fontan o Unión CavoPulmonar Total. En este caso se ilustra el conducto extracardiaco. A pesar que se han separado los circuitos venosos sistémico del pulmonar, y se habla que el paciente tiene una circulación en serie, aún quedan comunicaciones con paso de sangre desaturada hacia la circulación sistémica por la fenestración (F). (la pequeña abertura en el tubo que conecta la vena cava inferior a la arteria pulmonar, esta abertura permite el paso de sangre desaturada a la auricula unica) y por la sangre del seno coronario que también es sangre desaturada que llega a la aurícula única. Los pacientes tienden a saturar entre $82 \%$ y $95 \%$. El costo es una PVC elevada.

es responsable en 5-10 puntos porcentuales de disminución de saturación, por el shunt de derecha a izquierda a este nivel. El seno coronario sigue drenando en el atrio único y aporta con otros 5 a 7 puntos porcentuales de desaturación. Otras fuentes de sangre desaturada son las fístulas $A-V$ intrapulmonares residuales. Como verán, a pesar que el paciente retoma una circulación en serie, éste no satura $100 \%$, si no que lo hará en un rango de $82 \%$ a $95 \%$.

A continuación se mencionan las características fisiológicas y anatómicas de la cirugía de Fontan. (Tabla 16. Características de una cirugía de Fontan).

Los requerimientos para poder llevar a cabo y las ventajas de la operación de Fontan se describen en las Tablas 17 y 18 respectivamente.

Sin embargo, la operación de Fontan también presenta desventajas, entre las que se cuentan que se 


\section{Tabla 16. Características de una cirugía de Fontan}

\section{Características fisiológicas y anatómicas de la cirugía de Fontan}

Separación de circuitos sistémico y pulmonar: circulación en serie

Flujo pulmonar sin bomba impulsora

Shunt residual de derecha a izquierda:

- Cardiaco: Fenestración Fontan-Atrio y Drenaje del seno coronario al atrio

- Pulmonar: Fístulas A-V intrapulmonares.

Se eliminan sobrecargas de volumen (eliminadas en la etapa de Glenn)

Hipertensión venosa central crónica (grado variable)

\begin{tabular}{l}
$\begin{array}{c}\text { Tabla 17. Requerimientos para la cirugía de } \\
\text { Fontan }\end{array}$ \\
\hline Requerimientos para operación de Fontan \\
Resistencia pulmonar mínima \\
Árbol vascular pulmonar normal \\
Función ventricular adecuada \\
Que no exista insuficiencia de la válvula A-V \\
Ausencia de patología respiratoria \\
\hline
\end{tabular}

expone a una PVC elevada crónica, (lo que puede manifestarse por serositis, insuficiencia hepática y renal, enteropatía perdedora de proteínas y bronquitis plástica). Pueden producirse también trastornos del ritmo, (por distensión o cicatrices atriales), disminución de la capacidad funcional y disfunción ventricular tardía.

\section{Manejo anestésico del VU en etapa de Fontan}

Es importante revisar la historia clínica ya que en el seguimiento de estos paciente se debe buscar en forma dirigida signos de elevación de la PVC, (congestión yugular y hepática), realizar evaluación seriada de la función hepática, renal y respiratoria, búsqueda de trastornos del ritmo (arritmias atriales como Disfunción del Nodo Sinusal, fibrilación o flutter atrial, bloqueo de la conducción atrio-ventricular, etc.). Revisar las ecocardiografías seriadas para determinar la función ventricular y valvular, obstrucción del tracto de salida sistémico, la presencia de trombos, entre otras. También revisar el estado nutricional (por la enteropatía perdedora de proteínas).

Para un adecuado manejo anestésico se debe recordar que estos pacientes son dependientes de la Precarga. No toleran la hipovolemia (atención con ayunos preoperatorios prolongados sin aporte de volumen intravenoso). Se debe mantener una gradiente

\section{Tabla 18. Ventajas de la cirugía de Fontan}

Protección de la función ventricular en el largo plazo

Capacidad funcional adecuada

Flujo pulmonar variable según actividad

Minimiza el estigma de la cianosis profunda

Proporciona una calidad de vida aceptable

Racionaliza la necesidad de órganos para trasplante

transpulmonar sobre 10, es decir la presión en el árbol pulmonar debería ser a lo más 15 y ojalá menor a 20 $\mathrm{mmHg}$, esto es importante para la estrategia ventilatoria que debe evitar presiones ventilatorias elevadas. Es importante mencionar el caso de la cirugía laparoscópica, ya que ésta aumentará la presión intraabdominal, disminuyendo el retorno venoso sistémico de la VCl. Recordar que la comunicación entre cirujano y anestesista es crucial para usar la mínima presión intraabdominal posible. En todo caso existen numerosos reportes y experiencia de este tipo de cirugía en pacientes portadores de un Fontan.

Tener presente que si se instala un catéter venoso central en los vasos del cuello, la presión que se está midiendo es la presión de la arteria pulmonar. Si hay una resistencia vascular o presión pulmonar elevada, (sobre $20 \mathrm{mmHg}$ ), se recomienda hiperventilar, usar una $\mathrm{FiO}_{2}$ elevada, usar bicarbonato (para llevar el Ph $>7,5)$, eventualmente usar vasodilatadores pulmonares (milrinona, óxido nítrico). No debe descuidarse la función del ventrículo, por lo cual puede ser necesario usar un inótropo. La mejoría del inotropismo y disminución de la postcarga son beneficiosos para la función del VU, mantener el ritmo sinusal es muy importante. En el caso de existir una fenestración a nivel atrial, se debe tener presente que existe el riesgo potencial de una embolia aérea paradojal.

Respecto de los medicamentos anestésicos, se 


\begin{tabular}{|c|c|}
\hline \multicolumn{2}{|c|}{$\begin{array}{l}\text { Grilla de manejo del paciente con VU en etapa II } \\
\text { (Glenn) }\end{array}$} \\
\hline Contractilidad & Mantener \\
\hline F Cardiaca & Mantener (ritmo sinusal ideal) \\
\hline Precarga & Elevada \\
\hline RVS & Mantener \\
\hline RVP & $=0 \downarrow$ \\
\hline $\mathrm{FiO}_{2}$ & No es relevante \\
\hline
\end{tabular}

aplican los mismos preceptos que en un paciente portador de un GBD, prácticamente cualquier medicamento puede ser utilizado siempre y cuando se titulen cuidadosamente, se eviten los aumentos de la resistencia periférica y las maniobras que aumenten la resistencia vascular pulmonar. Los pacientes portadores de un Fontan suelen estar bajo tratamiento antiagregante plaquetario y/o anticoagulante, el que debe ser suspendido previo a una cirugía y reiniciarse prontamente luego del procedimiento. Respecto de la monitorización, si se trata de una cirugía menor puede utilizarse la monitorización estándar. Ante una cirugía mayor es conveniente utilizar monitorización invasiva, entre otros para realizar un control frecuente y seriado del estado acido-base, presión arterial y pre- sión venosa central. (Tabla 19. Grilla para el manejo del paciente portador de VU en etapa Fontan).

\section{Conclusiones}

Las cardiopatías congénitas son las malformaciones más frecuentes en la edad pediátrica. En la actualidad la mayor parte de ellas tiene una alternativa quirúrgica correctora o paliativa. Es probable que un anestesiólogo general pueda verse enfrentado a manejar pacientes portadores de una cardiopatía antes de su corrección, después de la corrección o eventualmente en etapas intermedias como es el caso del paciente portador de ventrículo único. La diversidad anatómicas de las distintas patologías hace complejo para el anestesiólogo general enfrentar a un paciente cardiópata. Es relevante conocer someramente la condición actual del paciente, tener una idea de la fisiopatología para plantear un objetivo hemodinámico apropiado para la patología en particular, y estar familiarizado con el efecto de las distintas drogas anestésicas y de las maniobras ventilatorias sobre el flujo sistémico y pulmonar, de manera de asegurar al paciente la condición hemodinámica más favorable a la patología subyacente. El articulo presenta un enfrentamiento práctico a cada patología basado en grillas de manejo hemodinámico que facilitarán al anestesiólogo general el enfrentamiento de un paciente en particular.

\section{Referencias}

1. Jenkins KJ, Gauvreau K, Newburger JW, Spray TL, Moller JH, lezzoni LI. Consensus-based method for risk adjustment for surgery for congenital heart disease. J Thorac Cardiovasc Surg. 2002 Jan;123(1):110-8.

2. Ramamoorthy $C$, Haberkern CM, Bhananker SM, Domino $\mathrm{KB}$, Posner KL, Campos JS et al. Anesthesia-related cardiac arrest in children with heart disease: data from the Pediatric Perioperative Cardiac Arrest (POCA) registry. Anesth Analg. 2010
May; 110(5):1376-82.

3. White MC, Peyton JM. Anaesthetic management of children with congenital heart disease for non-cardiac surgery. Continuing Education in Anaesthesia. Critical Care \& Pain J. 2012;12:17-22.

4. Baum VC, Palmisano BW. The immature heart and anesthesia. Anesthesiology. 1997 Dec;87(6):1529-48.

5. Allen U. Infective endocarditis: updated guidelines. Can J Infect Dis Med Microbiol. 2010;21(2):74-7.

6. Anesthetic agents and their cardiovascular effects. Chapter 6 . In Anesthesia for Congenital Heart
Disease. Andropoulos DB. $3^{\text {a Ed. }}$ 2015. Wiley.

7. Friesen $\mathrm{RH}$. Anesthetic drugs in congenital heart disease. Semin Cardiothorac Vasc Anesth. 2014 Dec;18(4):363-70.

8. Gottlieb EA, Andropoulos DB. Anesthesia for the patient with congenital heart disease presenting for noncardiac surgery. Curr Opin Anaesthesiol. 2013 Jun;26(3):318-26.

9. Donald DE, Essex HE. Pressure studies after inactivation of the major portion of the canine right ventricle. Am J Physiol. 1954 Jan;176(1):155-61. 